\title{
On Nonlinear Systems with Poorly Behaved Zero Dynamics
}

\author{
F. Doyle III F. Allgöwer* S. Oliveira E. Gilles* M. Morarit \\ Chemical Engineering 210-41, California Institute of Technology, Pasadena, CA 91125
}

\begin{abstract}
The design of controllers for nonlinear, nonminimum-phase systems is very challenging and is currently considered to be one of the most difficult theoretical control problems. Most control algorithms for nonlinear procesees perform a linearization making use of an inverse of the system. In the linear case, the system can be factored into the minimum-phase and the nonminimum-phase parts and only the first one is inverted for purposes of control design. A similar scheme for nonlinear systems is still under investigation.

The preaent work adreses the problem of synthesizing nonlinear state feedback controllers for nonlinear, nonminimum-phase processes in three different ways. The first approach consists of a partial linearization which preserves stability by using an approximate stable/anti-stable factorisation. The second technique can be viewed as an inner-outer factorization based approach. And, finally, in the single-output case, it is ahown (through an example) that stabilization of the internal dynamics of a nonminimum-pluase system can be achieved by using an additional input if this is feasible in practice. In this case, the manipulated variables have different roles, i.e., one is chosen such as to input/output feedback linearize the system and the second is used to locally stabilize the resulting nonminimum-phase internal dynamics.
\end{abstract}

\section{Introduction}

It is widely recognized that a controller must explicitly or implicitly generate a process inverse. Methods with explicit inversion include internal model control [5] and the generalized Smith predictor [11]. When dealing with nonminimum-phase systems, a stable/unstable decomposition is necessary and the controller must invert only the part with stable inverse. These approaches are roughly equivalent (under the assumption of a perfect model) to controlling the corresponding minimum-phase part of the system, leaving the nonminimum-phase component in open-loop.

In the nonlinear case, the problem of constructing control algorithms for processes with unstable inverses is equally important, since the available algorithms also rely either implicitly (see, e.g., [8]) or explicitly (see, e.g., [3]) on generating an inverse of the process. The main difficulty here is that results on decomposition into minimum-phase and nonminimum-phase portions are available only for second-order systems (see [9]).

Recently, Wright and Kravaris ([13]) presented an approach to deal with nonlinear, nonminimum-phase systems based on the notion of statically equivalent outputs; a minimum-phase statically equivalent output is estimated on-line and then an available nonlinear control algorithm is used to control it to the set point. Therefore, instead of looking for a decomposition of the process dynamics, this formulation is based on the calculation of a suitable output function.

One assumption is central in all predictor-type approaches and will also be necessary in this work. The system under consideration is assumed to be open-loop stable.

Throughout the paper, we will consider nonlinear systems affine in the input variables

$$
\begin{aligned}
& \dot{z}=f(x)+g(x) u, \\
& y=h(x), \quad x \in \mathfrak{\Re}^{*}, u \in \mathfrak{\Re}^{m}, y \in \mathfrak{R}^{P}
\end{aligned}
$$

\section{Stable/Anti-stable Factorization Approach}

\subsection{Motivation}

Because controllers resulting from linearization schemes generate an inverse of the process, nonminimum-phase systems do not adnit exact input/output linearization with internal stability. However, one can try to linearize as much of the nonlincarities of the system as possible under the constraint that internal stability is preserved. One way of approaching this problem involves an approximate stable/anti-stable factorization of the zero dynamics of the system. This is the technique presented in this section.

2.2 Technique

First system (1) is transformed into normal form (see, e.g., [7]) through a nonlinear change of coordinates $\left(z_{i}=\phi_{i}(x), i=1, \ldots, n\right)$. The resulting system in the transformed varibles is then written as:

$$
\begin{aligned}
\dot{z}_{i} & =z_{i+1}, \quad i=1, \cdots, r-1 \\
\dot{z}_{r} & =a(\xi, \eta)+b(\xi, \eta) u
\end{aligned}
$$

\footnotetext{
"Institut fur Syetemdynamik \& Regelungstechnik, Universität Stuttgart, Germany

tTo whom correopondence should be addressed: phone (818)3.56-4186, fax (818)568-8743, e-mail mmeimc.caltech.edu
}

$$
\begin{gathered}
\dot{\eta}=c(\xi, \eta) \\
y=z_{1}
\end{gathered}
$$

where $\xi$ and $\eta$ are standard notations for the two sets of coordinates, of dimensions $r$ and $n-r$, respectively. Thus

$$
\boldsymbol{\xi}=\left[\begin{array}{c}
z_{1} \\
\vdots \\
z_{r}
\end{array}\right] \text { and } \eta=\left[\begin{array}{c}
z_{r+1} \\
\vdots \\
z_{n}
\end{array}\right]
$$

In this form, the $(n-r)-$ dimensional subsystem given by

$$
\dot{\eta}=c(\xi, \eta) \text {, }
$$

is completely unobeervable and therefore called the internal dyuamics of the system. If (3) is locally stable, the standard static control law

$$
u=\frac{v-a(\xi, \eta)}{b(\xi, \eta)}
$$

linearizes the input/output map of the system witl $\frac{(\cdot)}{v(0)}=H(s)$. However, if the internal dynamics is unstable, this approach does not work because some of the unobservable modes of the system become unstable when linear input/output behavior is imposed. In order to solve this problem one can try to establish a compromise between making the input/output map "as linear as possible" and some portion of the original intermal dynamics observable in order to achieve internal stability. Thus, instead of (4), one can think of using a feedback control law of the form

$$
u=\frac{1}{b}(-a-r(\xi, \eta)+v)
$$

where $r(\xi, \eta)$ is a general function of the transformed variables chosen such as to eliminate as much of the nonlinearities in the input/output channel as possible under the constraint that the system

$$
\begin{gathered}
\dot{z}_{1}=z_{2} \\
\vdots \\
\dot{z}_{r-1}=z_{r} \\
\dot{z}_{r}=-r(\xi, \eta)+v \\
\dot{\eta}=c(\xi, \eta)
\end{gathered}
$$

is stabilizable.

An improved way of approaching this problem is to make only the unstable modes of the internal dynamics observable. In order to do this we should first be able to factorize the internal dynamics into stable and anti-stable parts and leave only the stable component unobservable. In general, this factorization cannot be exactly performed. However, an approximate factorization approach can be carried out as follows.

Once we have the system in its normal form, a diffeomorphic transformation

$$
T:\left\{\begin{array}{l}
\eta_{1}=T_{1}(\eta) \\
\eta_{2}=T_{2}(\eta)
\end{array}\right.
$$

is performed, factorizing the undriven internal dynanics (or zero dynamics)

$$
\begin{array}{lc}
\text { into an anti-stable part } & \dot{\eta}=c(0, \eta) \hat{\oplus} c^{*}(\eta) \\
& \dot{\eta}_{1}=c_{1}^{*}\left(\eta_{1}\right)
\end{array}
$$

and a stable part (when viewed as a driven system)

$$
\dot{\eta}_{2}=c_{2}^{*}\left(\eta_{1}, \eta_{2}\right)
$$

where $\eta_{1}$ is the unstable manifold coordinate and $\eta_{2}$ is a coordinate independent of $\eta_{1}$

Remark 1: It is not necessary that the transformation $T$ decomposes the system into its anti-stable and stable parts. It is sufficient for stabilization purposes to get the anti-stable part correctly. Therefore, the calculation of $T$ requires only construction of the local stable manifold $W_{l o c}^{i}(0)$.

System (2) with transformation $T$ leads to:

$$
\begin{gathered}
y=z_{1} \\
\dot{z}_{1}=z_{2} \\
\dot{z}_{2}=z_{3} \\
\vdots \\
\dot{z}_{r-1}=z_{r} \\
\dot{z}_{r}=\bar{a}\left(\xi, \eta_{1}, \eta_{2}\right)+\bar{b}\left(\xi, \eta_{1}, \eta_{2}\right) u
\end{gathered}
$$


with

$$
\dot{\eta}_{2}=c_{2}\left(\xi, \eta_{1}, \eta_{2}\right)
$$

$$
\begin{aligned}
& c_{1}\left(\xi, \eta_{1}, \eta_{2}\right)=\left.\frac{\partial T_{1}}{\partial \eta} c(\xi, \eta)\right|_{q=T^{-1}\left(\eta_{1}, \eta_{2}\right)} \\
& c_{2}\left(\xi, \eta_{1}, \eta_{2}\right)=\left.\frac{\partial T_{2}}{\partial \eta} c(\xi, \eta)\right|_{7=T^{-1}\left(\eta_{1}, \eta_{2}\right)}
\end{aligned}
$$

Therefore, in order for the stable part to have no influence on the unstable one, the following condition

$$
\frac{\partial}{\partial \eta_{2}}\left(\left.\frac{\partial T_{1}}{\partial \eta} c(\xi, \eta)\right|_{\eta=T-1}\left(\eta_{1}, \eta_{2}\right)\right)=0
$$

must hold everywhere. In general, this will not be the case. However $\eta_{2}$ always enters the expression for $\dot{\eta}$ only in terms of order greater than or equal to $q=2$. This minimal crose-over order is intrinsic to the system and cannot be changed. Therefore, the unatable zero dynamics can be thought of as having a part of order less than that does not depend on $\eta_{n}$ and higher order terms depending on $\xi, \eta_{1}, \eta_{2}$, i.e.

with $\alpha+\beta+\gamma=q$

$$
\dot{\eta}_{1}=\overline{c_{1}}\left(\xi, \eta_{1}\right)+\mathcal{O}\left(\xi^{\alpha} \eta_{1}^{\beta} \eta_{2}^{\gamma}\right)
$$

The application of the feedback

$$
u=\frac{1}{b}\left(-\bar{a}-r\left(\xi, \eta_{1}\right)+v\right)
$$

where $r\left(\xi, \eta_{n}\right)$ is chosen such that the system

$$
\begin{gathered}
y=z_{1} \\
\dot{z}_{1}=z_{2} \\
\dot{z}_{2}=z_{3} \\
\vdots \\
\dot{z}_{r-1}=z_{r} \\
\dot{z}_{r}=-r\left(\xi, \eta_{1}\right)+v \\
\dot{\eta}_{1}=\bar{c}_{1}\left(\xi, \eta_{1}\right)+O\left(\xi^{\alpha} \eta_{1}^{\beta} \eta_{2}^{\gamma}\right) \\
\dot{\eta}_{2}=\bar{c}_{2}\left(\xi, \eta_{1}, \eta_{2}\right)
\end{gathered}
$$

is stabilizable when terms of order $q$ and higher in the expression for $\dot{\eta}_{1}$ are neglected.

Thus, if we only consider terms of order up to $q-1$, the unobservable part of the internal dynamics is stable.

In many cases, the linear approximation can be made stabilizable with a linear feedback term

$$
r\left(\xi, \eta_{n}\right)=p^{T} \xi+s^{T} \eta_{1}
$$

$s$ and $p$ being constant vectors of appropriate dimensions, when the linear approximation of the original system is controllable.

Using a nonlinear dependency of $r$ on $\eta_{1}$ and $\xi$, the remaining nonlinearities in the input/output channel can be influenced. Exact guidelines on how to choose $r(\xi, \eta)$ are however not easy to obtain and there is no guarantee that there will always exist a function $r\left(\xi, \eta_{1}\right)$ such that the system can be stabilized. If no such a $r\left(\xi, \eta_{1}\right)$ exists that renders the resulting system (9) stabilizable, one can also try to make a part of the stable internal dynamics observable in order to achieve stabilizability.

Remark 2: We assumed so for that it is possible to find a tmanformation to factorize the zero dynamics globally into stable and enti-stable parts. As our accuracy can never exceed onder $q$, it is sufficient for stability to locally decompose the uxdriven internal dynamics up to order $q$. This can alvays be done using similar technigues as in the center manifold theory (see, e.g., $[1,12]$ ). It should however be stressed that, in order to find the exact q, not only a lower bound on the actwal $q$, we need to know the stable/antistable transformation. Therefore, this has to be done in an iterative manner.

Remark 3: By wing this technigue, we get a partially linearized system that is at leest locally stable. However, no gearantee can be given that the resulting system is less nonlinear. It is, in general, a nonlinear system of lower order and in many cases the term $r(\xi, \eta)$ can be chosen so as to minimize the effect of nonlinearities in the inpat/output channel.

2.3 Example

Let us consider the following simple example to motivate the stable/ anti-stable factorization approach to the control of nonminimumphase systems:

$$
\begin{gathered}
\dot{x_{1}}=x_{2} \\
\dot{x_{2}}=-x_{1}-2 x_{2}+x_{3}+10 x_{4}+x_{3} x_{4}+u \\
\dot{x_{3}}=-2 x_{1}-3 x_{2}+x_{3} \\
\dot{x_{4}}=-x_{4}+0.3 x_{2}^{2} \\
y=x_{1}
\end{gathered}
$$

This is an open-loop stable system with strongly nonlinear input/output behavior and nonminimum-phase properties. This system is already in its normal form and we can easily identify that the last two equations constitute the internal dynamics. Moreover, $x_{3}$ is the unstable mode of the internal dyramics and $x_{1}$ the stable one. Note that the stable and the unstable parts of the zero dynamics is already completely decoupled.

By using standard control theory, this system cannot be feedback linearized. Bowever, the following feedbeck control law:

$u=x_{1}+2 x_{2}-x_{3}-10 x_{4}-x_{3} x_{1}-p_{1} z_{1}-p_{2} x_{2}-s_{1} x_{3}+v$

exactly linearizes the input/output map and permits the resulting system to achieve internal stability simultaneously. The interesting feature about this simple example is that because the unstable portion of the internal dynamics is linear, it is possible to exactly linearize the input/output map and achieve internal stability at the same time. Howeves, the standard input/output linearization approach is not able to find such a stabilizing control law.

By choosing $p_{1}=3.6, p_{2}=7, s_{1}=-4.8$, the closed-loop poles of the linearized system are $\lambda_{1}=-1, \lambda_{2}=-2, \lambda_{3}=-3, \lambda_{1}=-1$.

Simulations of the output behavior for the original system and its linearized version for step changes in the input variables ( $u$ and $v$, respectively) are presented below:

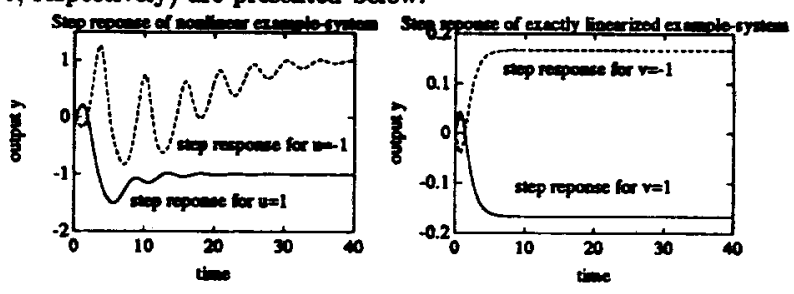

3.1 Motivation

3 Inner-Outer Factoration Approach

We have mentioned that exact linear behavior of the input/output map can only be achieved for systems with well-behaved internal dynamics. Thus, the important question to be adressed is the systematic degradation of the linear input/output map in order to achieve stable internal dynamics.

The previous section introduced a stable/anti-stable factorization appraach to accomplish this task. In this section, we seek an inner-outer factorization-based approach.

3.2 Technique

The main objective here is, given a nonlinear dynamical system $(P)$ with unstable zero dynamics, to derive a minimum-phase nonlinear system $\left(\boldsymbol{P}_{M}\right)$ with the following properties:

(i) Poles of the linearization of $P$ around a given equilibrium point $=$ Poles of the linearization of $\boldsymbol{P}_{\boldsymbol{M}}$ around the same point (along the whole equilibrium manifold),

(ii) Zeros of the linearization of $P$ around a given equilibrium point $=$ "reflection" of the zeros of the linearization of $P_{M}$ around the same point (along the whole equilibrium manifold)

(iii) Static gain of $P=$ Static gain of $P_{M}$.

From (ii) we can see immediatly that this technique requires all zeros of the linearization of $P$ around the equilibrium point to be in the RHP (i.e., the system has to be maximally nonminimum-phase). This restriction comes from the fact that this approach reflects all the zeros of $\boldsymbol{P}$ when constructing the minimum-phase system $\boldsymbol{P}_{\mathbf{Y}}$.

Here we will not use the normal form of nonlinear systems in the sense of Byrnes/Isidori (2) because, by manipulating $c(\xi, \eta)$ we will not only affect the zero dynamics but also the pole dynamics, which we do not want to disturb since the plant is assumed to be open-loop stable. We will make use of the Fliess canonical form [4] or Zeitz/Krener observability canonical form $[14,10]$, whose interesting feature is that the pole and zero dynamics manifest themselves separately:

$$
\begin{gathered}
\dot{z}_{1}=z_{2} \\
\vdots \\
z_{m-1}=z_{m} \\
\dot{z}_{m}=F\left(z, u, \dot{u}, \ldots, u^{(n-r)}\right)
\end{gathered}
$$

In this case, feedback linearization can be achieved by dynamic compensation of the form:

$$
F\left(z, u, \dot{u}, \ldots, u^{(n-r)}\right)=\sum_{i=1}^{\infty} \dot{\beta}_{i} z_{i}+v
$$

where the coefficients $\hat{\beta}_{i}$ are chosen in the usual way (to achieve desired closed-loop performance). obtain:

By performing a Jacobian linearization of the system (11) we

$$
\dot{z}_{1}=z_{2}
$$




$$
\begin{gathered}
z_{n-1}=z_{n} \\
\text { where } \\
\alpha_{1} z_{1}+\alpha_{2} z_{2}+\ldots+\alpha_{n} z_{n}+\beta_{0} u+\beta_{1} \dot{u}+\ldots+\beta_{n-,} u^{(n-r)} \\
\alpha_{i}=\frac{\partial F}{\partial z_{i}} l_{\text {eq, }} \\
\beta_{i}=\frac{\partial F}{\partial u(i)} l_{\text {eq }},
\end{gathered}
$$

Note that the tranfer function for (13) is given by:

$$
G^{*}(s)=\frac{y(s)}{u(s)}=\frac{\beta_{0}+\beta_{1} s+\ldots+\beta_{n-r} s^{n-r}}{\alpha_{1}+\alpha_{2} s+\ldots+\alpha_{n} s^{n}}
$$

The construction of the minimum-phase system $P_{M}$ is performed by application of time reverall to the derivatives of 4 (a procedure that affects only odd powered derivatives) in order to schieve the "reflection" of the zeros through time $t=0$. The key point here is that any of the terms appearing in the calculation of the coefficients $\beta_{i}$ (which determine the seros of (14)) will influence the calculation of the coefficients $\alpha_{i}$ (which determine the pole dynamics of (13)).

To make the idea of time seversal clearer, let us recall the definitions of local atable and unstable manifolds in the neighborhood of a fixed point $z\left(W_{i \infty}^{i}(z), W_{l o c}(z)\right)$ :

$W_{l \infty}^{\prime}(\bar{x})=\left\{x \in U \mid \phi_{t}(x) \rightarrow \bar{z}\right.$ as $\left.t \rightarrow \infty ; \phi_{t}(x) \in U \forall t \geq 0\right\}$

$W_{l o c}^{\infty}(\bar{x})=\left\{x \in U \mid \phi_{t}(x) \rightarrow\right.$ as $\left.t \rightarrow-\infty ; \phi_{l}(x) \in U \forall t \leq 0\right\}$

where $U \subset x^{n}$ is a neighborhood of $\bar{x}$ and $\phi_{t}(x)$ is the flow of the dynamic system.

Therefore, we notice from these definitions that the spans of the stable and unstable eigenvectors can be "interchanged" by time reversal.

This means that $P_{M}$ is, by construction, a (locally) minimumphase system.

The fundamental ides applied to the derivation of $P_{M}$ is analo gous to the inner-outer factorization of linear systems, in which the transfer function of the system $(G)$ is factorized into:

$G_{M}$ : minimum-phase (invertible) part; unstable zeros of $G$ appear "reflected" through the imaginary axis in $G_{M}$.

$G_{A}$ : stable part; "all-pass"; unity dynamic gain; its poles are equal to the "refiected" zeros of $G$.

such that, $G=G_{M} \cdot G_{A}$.

Thus we can see the "reflection" of the unstable zeros of $G$ across the $j \omega$-axis to yield poles of $G_{A}$ and zeros of $G_{M}$ as a symmetry in time.

The resulting dynamic control law is determined by the solution of the following ordinary differential equation along trajectories of the cloeed-loop system:

$$
\begin{aligned}
F_{M}\left(z, u, \dot{u}, \ldots, u^{(n-r)}\right) & \doteq \\
F\left(z, u,-\dot{u}, \bar{u},-u^{(3)}, \ldots, u^{(n-r)}\right) & =\sum_{i=1}^{n} \hat{\beta}_{i} z_{i}+v
\end{aligned}
$$

One of the advantages of the construction of $P_{M}$ from $P$ is that the calculation is straightforward and, aside from the maximum nonminimum-phase condition on $P$, no more restrictions are present.

Other important characteristics of this innes-outer factorization procedure are:

(i) The derivation is in the same spirit as the Hauser approximate linearization appraaches (neglecting/odding terms to higher order derivatives of $y$ ) [6].

(ii) The derivation draws on tools from differential algebre which appears to be a more attractive framework for system inversion, realization, etc [4].

(iii) In terms of complexity of the calculations, the only burdle is the inversion of the control dependent coordinate transformation. Other approaches for control of nonminimum-phase systems $([13,9])$ involve at least the same level of complexity in the calculation of their so-called "natural coordinates". In particular, [13] also requires the system to be involutive.

(iv) Although we restrict ourselves to maximally nonminimum-phase systems in this report, the possibility that we could use a stable/ anti-stable factorization (the technique described in the previous section) on the differential representation of the plant and then perform the time inversion on the unstable part of the zero dynamics may not be discarded.

Note that this approach provides a minimum-phase portion of the system but does not actually factor $P$ into nonlinear minimumphase and all-pass components. This is not as critical as it seems, however, because for control design, we are primarily interested in the minimum-phase portion itself.

Since [9] introduces a class of static feedback laws that makes the closed-loop system equivalent, under appropriate coordinate transformations, to a nonlinear first-order all-pass in series with a linear first-order lag, we found it useful to examine the equivalence between these and our results (in the case of two-dimensional systems). We concluded that the dynamics of the unobservable part of the system in the two cases are not identical (although they can be related by a diffeomorphism and share local stability properties). Kravaris and Deoutidis seek an outer factor that has the same dynamics as the plant and only a different output map. This makes the nonlinear ISE-optimization problem easier. In the present rork, the outer factor has dramatically different dynamics (in general, not control affine). This makes the nonlinear ISE-optimization problem somewhat more difficult.

The plant $\boldsymbol{P}_{M}$ approximates (to some degree) the original nonlineas plant $P$. Furthermore this "approrimation" becomes the outer factor (from inner-outer factorization) in the limit of linear behavior.

This suggests a control architecture like the Smith Predictor for linear plants. The following block diagram illustrates the way the control action, given by solving equation (15) along trajectories of the closed-loop system, is implemented:
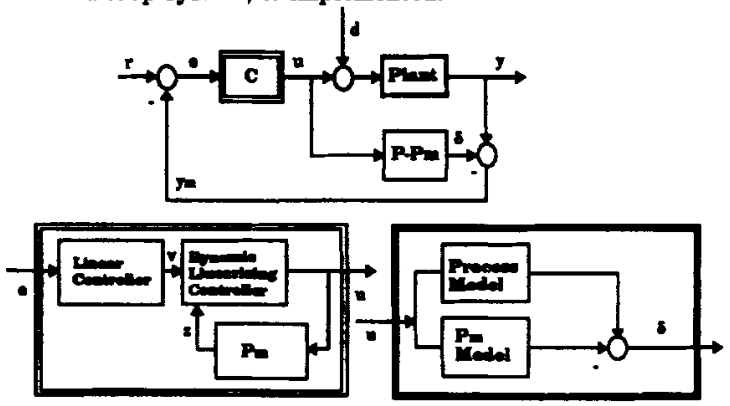

Controller C Minimu Phase Correction Signal

Here $\boldsymbol{P}_{\mathbf{M}}$ is the outer factor which has the property that, along the equilibrium locus, it yields an output equivalent to that of $P$ (i.e., they are statically equivalent). Thus, tracking of $y$ is guaranteed by tracking of $y_{M}$. The controller, $C$, is the standard input/output linesrizing controller (designed for $\boldsymbol{P}_{M}$ ). We notice that an openloop observer is required.

3.3 Example

Let us consider a CSTR reactor with isothermic Van de Vusee kinetics and a time delay in the output measurement (apporimated with a Padé approximation). Here we will look at a first order Padé approximation, but note that any other approximation is maximally nonminimum-phase as well.

$$
\begin{gathered}
\dot{x}_{1}=-k_{1} x_{1}-k_{3} x_{1}^{2}+u\left(x_{10}-x_{1}\right) \\
\dot{x_{2}}=k_{1} x_{1}-k_{2} x_{2}+-u x_{2} \\
\dot{x_{3}}=\frac{-2}{t_{d}} x_{3}+\frac{4}{t_{d}} x_{2} \\
y=x_{3}-x_{2}
\end{gathered}
$$

Straightforward calculations lead to:

$$
\begin{aligned}
y^{(i i i)} & =F(x, u, \dot{u}, \bar{u})=a(x)+b(x) u+c(x) u^{2}+d(x) u^{3} \\
& +e(x) \dot{u}+f(x) u \dot{u}+g(x) \bar{u}
\end{aligned}
$$

where $x=\left[x_{1}, x_{2}, x_{3}\right]^{T}$.

Therefore, for dynamic feedback linearization, we should synthetize $v$ such that:

$$
F(x, u, \dot{u}, \tilde{u})=-\alpha_{1} y-\alpha_{2} \dot{y}-\alpha_{3} \bar{y}+k v
$$

where the parameters $k$ and $\alpha_{i}, i=1,2,3$, are designed for closedloop performance. However, because this is a purely nonminimumphase system, the above synthesis would lead to an unstable controller. Thus, instead of designing the controller based upon $F(x, u, \dot{u}, \bar{u})$, we design it based upon $F_{M}(x, u, \dot{u}, \bar{u}) \doteq F(x, u,-\dot{u}, \bar{u})$.

In terms of practical implementation, there is a further complication: we need to construct an open-loop observer in order to reconstruct the state variables (as mentioned before).

So, there are two pieces to the controller design, i.e., generating $u$ (dynamic compensator) and generating the transformed states $\left(z_{i}, i=1,2,3\right)$, for the dynamic compensator to use. For the estimator of the states, we employ an open-loop observer requiring $u, \dot{u}, \bar{u}$ as inputs. Additionally, for the dynamic compensator, we define the following (transformed) $F_{M}$ function $\left(\bar{F}_{M}\right)$ :

$$
\begin{array}{r}
\hat{F}_{M}(z, u, \dot{u}, \bar{u})=\hat{a}(z, u, \dot{u})+\dot{b}(z, u, \dot{u}) u+\dot{c}(z, u, \dot{u}) u^{2} \\
+\dot{d}(z, u, \dot{u}) u^{3}+\hat{e}(z, u, \dot{u}) \dot{u}+\hat{f}(z, u, \dot{u}) u \dot{u}+\dot{g}(z, u, \dot{u}) \dot{u}
\end{array}
$$

Then, the resulting dynamical controller (with states $u_{0}, u_{1}$ ), is given by:

$$
\begin{gathered}
\dot{u}_{0}=u_{1} \\
\dot{u_{1}}=\frac{\left[\left(-\alpha_{1} z_{1}-\alpha_{2} z_{2}-\alpha_{3} z_{3}\right)+k v\right.}{\grave{g}}
\end{gathered}
$$




\section{$-\frac{\left.\left(\hat{a}+\hat{b} u_{0}+\hat{c} u_{0}^{2}+\hat{d} u_{0}^{3}+\hat{c} u_{1}+\hat{f} u_{0} u_{1}\right)\right]}{u=u_{0}^{\hat{g}}}$}

\section{Multiple-Inpat Approach}

4.1 Motivation

We know that if system (1) bas relative degree $r$ at a point $x_{0}$, it is always poesible to find a nonlinear coordinate transtormation that puts the system in the so-called normal form (in the sense of Byrnes/lsidori) (2). Then it is eary to see that the state feedback

$$
u=\frac{v-a(\xi, \eta)}{u(\xi, \eta)}
$$

will transform the system into a syotem whose input/output behavior is identical to that of a linear system having a transfer function

$$
\frac{v(s)}{v(s)}=H(s)=\frac{1}{s}
$$

As in the normal form $y=z_{1}$, aymptotic tracking of the output may be achieved by selecting the external input $v$ auch that the matrix aesociated with the first r-dimentional system in (2) has all eigenvalues in the LHP. However, this procedure will work only in the case that the internal dynamics of (2), given by

$$
\dot{\eta}=c(\xi, \eta)
$$

are locally stable (i.e., in a neighborhood $U$ of the point $x_{0}$, all the eigenvalues of the linearization of $\dot{\eta}=c(0, \eta)$ lie in the LHP).

If the zero dynamics is unstable one can take advantage of additional manipulated variables if available. While some of these variables are used to linearize the input/output map, others may be used to locally stabilize the internal dynamies (which remain nnobservable but can now be made stabilizable).

4.2 Teehnique

To make the explanation clearer, let us consider the single-output case (the extension to MIMO systems is straightforward). Let us suppoee that with respect to a certain input $u_{1}$, the syatem bas a well-defined relative degree $r$ at the point $x_{0}$ (the operating point around which we desire to operate the system, for example).

Remark: By well-defined relative degree we mean that the point $x_{0}$ may not be a singularity. So far, we have identified two different types of singular points, at which the static control law resulting from the input /oxtput linearization procedsre described above is not well-defined. In the firt ase, the gain of the binearization of the surtem changes sign at the siagular point and, in the second, one or more zeros move across the jw-axis. In both cases, we lose controllability for ell prectical purposes.

Let us assume that the obtained zero dynamics is unstable (one or more eigenvalues of its linearization around $x_{0}$ has a non-negative real part). If there is apother manipulated variable in the real system, then we can consider an extra feedback control law $u_{2}$ that will permit us to locally stabilize the zero dynamics. This means that though the zero dynamics is completely undriven with respect to the input $u_{1}$ it can have its stability characteristics changed by $u_{2}$

Of course, this approach is justified only if all the poesible manipulated rariables with respect to which the system has well-defined relative degree at the operating point of interest, considered separately for purposes of input/output linearization, generate nonminimum-phase zero dynamics.

If we choose $u_{1}$ (input with respect to which the system has relative degree $r$ at $x_{0}$ ) to linearize the input/output behavior, we obtain the following "modified" normal form:

$$
\begin{gathered}
\dot{z}_{1}=z_{2} \\
\dot{z}_{2}=z_{3} \\
\vdots \\
\dot{z}_{r-1}=z_{r} \\
z_{r}=a\left(\xi, \eta, u_{2}\right)+b\left(\xi, \eta, u_{2}\right) u_{1} \\
\dot{\eta}=c\left(\xi, \eta, u_{3}\right) \\
y=z_{1}
\end{gathered}
$$

Then the next step is the selection of $u_{2}=u_{2}(\eta)$, such that the linearization of $\dot{\eta}=c\left(0, \eta_{1} u_{2}\right)$ at $x_{0}$ has all eigenvalues in the LHP. 4.3 Example

The example we will consider bere is a CSTR reactor with exothermic Van de Vusse kinetics (see [2] for the formulation of the isothermal case):

$$
\begin{aligned}
& \text { Model for the System: } \\
& \dot{x_{1}}=-k_{1}\left(x_{3}\right) x_{1}-k_{3}\left(x_{3}\right) x_{1}^{2}+u_{1}\left(x_{10}-x_{1}\right) \\
& \dot{x_{2}}=k_{1}\left(x_{3}\right) x_{1}-k_{3}\left(x_{3}\right) x_{2}-u_{1} x_{2} \\
& \dot{x_{3}}=\frac{-\Delta H_{1} k_{1}\left(x_{3}\right) x_{1}-\Delta H_{2} k_{3}\left(x_{3}\right) x_{2}-\Delta H_{3} k_{3}\left(x_{3}\right) x_{1}^{2}}{\rho C p}
\end{aligned}
$$$$
\begin{aligned}
A \stackrel{k_{1}}{\rightarrow} B \stackrel{k_{2}}{\rightarrow} C \\
2 A \stackrel{k_{3}}{\rightarrow} D
\end{aligned}
$$

$$
\begin{gathered}
+\frac{u_{1}\left(x_{30}-x_{3}\right)+z}{\rho C p} \quad y=x_{2}
\end{gathered}
$$

where $x_{1}$ and $x_{2}$ are the concentrations of $A$ and $B$, respectively, $x_{3}$ is the temperature of the reactor, $u_{1}$ is the flow rate $(F / V), u_{2}$ is the heat exchanged between the reactor and the surrounding $(Q), x_{10}$ is the concentration of $A$ in the feed atream, $x_{30}$ is the temperature of the feed stream and $k_{i}(x)=k_{i 0}-\frac{s_{i}}{k_{i}}, i=1,2,3$.

Now let us asoume that both the flow rate $y_{1}$ and the beat input to the reactor $u_{2}$ can be choeen as manipulated variables. We know that linearization of the inpot/outpat map can be performed by using a feedback law of the form:

$$
u=\frac{1}{L_{s} L_{j}^{n-1} h(x)}\left(-L_{j}^{n} h(x)+v\right)
$$

In our system, the relative degree of $u_{1}$ is equal to 1 and can be defined on the whole phase plane, since $L_{g_{1}} h(z)=-x_{2} \neq 0$ (where $f, g_{1}, g_{2}$ are defined according to the general format (1)). Therefore the system has well-defined relative degree $(r=1)$ with respect to $u_{1} \forall x \in x^{3}$. However, if we choose to linearize the input/output behavior of the system by using $v_{1}$, the resulting zero dymamics is nonminimum-phase.

So, let us consider the possibility of using $u_{2}$ in order to linearize the input/output map of (20). As

$$
K(x) \doteqdot L_{m} L_{f} h(x)=\frac{1}{V \rho C_{p}} \frac{\partial}{\partial x_{3}}\left[k_{1}\left(x_{3}\right) x_{1}-k_{2}\left(x_{3}\right) x_{2}\right],
$$

we conclude that the relative degree of the system with respect to $u_{2}$ is not defined everywhere (i.e., $r \neq 2$ at the points for which $K(x)=0$ ). This should not be a problem if the operating point we select is not one of the points at which $K$ vanishes identically.

Now suppose it is desired to operate the reactor at the point where $B$ reaches its maximum conversion on the equilibrium curve (which we will call optimum point and denote by $x^{*}$ ), i.e.,

$$
\frac{\partial x_{2}}{\partial x_{3}}\left(x^{*}\right)=0
$$

Thus we are interested in operating at the point for which the gain between the output $y=x_{2}$ and $x_{3}$ vanishes. Since $u_{2}$ is the heat input to the resctor and the gain between the concentration of $B$ (output variable) and the temperature is zero at the optimum, intuition suggests that the use of $u_{2}$ to control the system will be problematic.

We can easily show that $K\left(x^{*}\right) \neq 0$, i.e., the gain of the linearizstion of the system does not vanish at the optimum, which indicates that the singular locus does not intersect the equilibrium manifold at $x^{*}$. However, because the only zero of the linearization of the system moves from the LBP to the RHP through infinity, we have controllability problems at this point. Actually, at $z^{*}$, the finite zero of the system disappears. Due to this peculiar behavior of the system at $x^{*}$, this point is considered to be a singular point.

Note that the kind of singularity that the optimum point represents in this example is different from the singularity that we find in the case of a CSTR reactor with the exothermic kineties $A \rightleftharpoons B$ (for a more detailed discussion of this example see [3]). In this last case, the $\mathrm{g}$ ain is zero and simultaneously the zero of the linearization of the system disappears at the optimum point $\left(K^{\prime}\left(x^{*}\right)=0\right)$.

Thus, by using $u_{1}$ to linearize the input/output behavior we get a nonminimum-phase zero dynamics and by using $u_{2}$ for the same purpose we get a not well-defined relative degree at the optimum operating point (which means controllability problems). In order to illustrate the resulting behavior in this last case when we try to make the conversion of $B$ aymptotically approach its maximum value on the equilibrium curve, we have performed some numerical simulations of the system for the given set of parameters: $u_{1}=7$, $\Delta H_{1}=-5, \Delta H_{2}=-15, \Delta H_{3}=-20, E_{1}=15, E_{2}=25$, $E_{3}=15, k_{10}=1 e 8, k_{20}=1 e 10, k_{30}=1 e 7, x_{10}=10, x_{30}=300$, $p C p=1, V=1$. Notice that we have redefined the variables in terms of deviation variables with respect to the coordinates of the optimum point. The external linearizing control variable o was selected such that the matrix associated with the linearization of the system around $x^{*}$ has one real negative eigenvalue and a pair of complex conjugates with negative real part. Thus the kind of behavior we wish to obtain is that for initial conditions sufficiently close to the origin, the control $u_{3}$ is able to drive the system towards the origin.

However, we can clearly see in the plots shown below that despite the huge magnitude of $u_{2}$, the heat removed from the reactor, trajectories starting very close to the origin do not approach the origin and tend to behave in a very unpredictable way. 

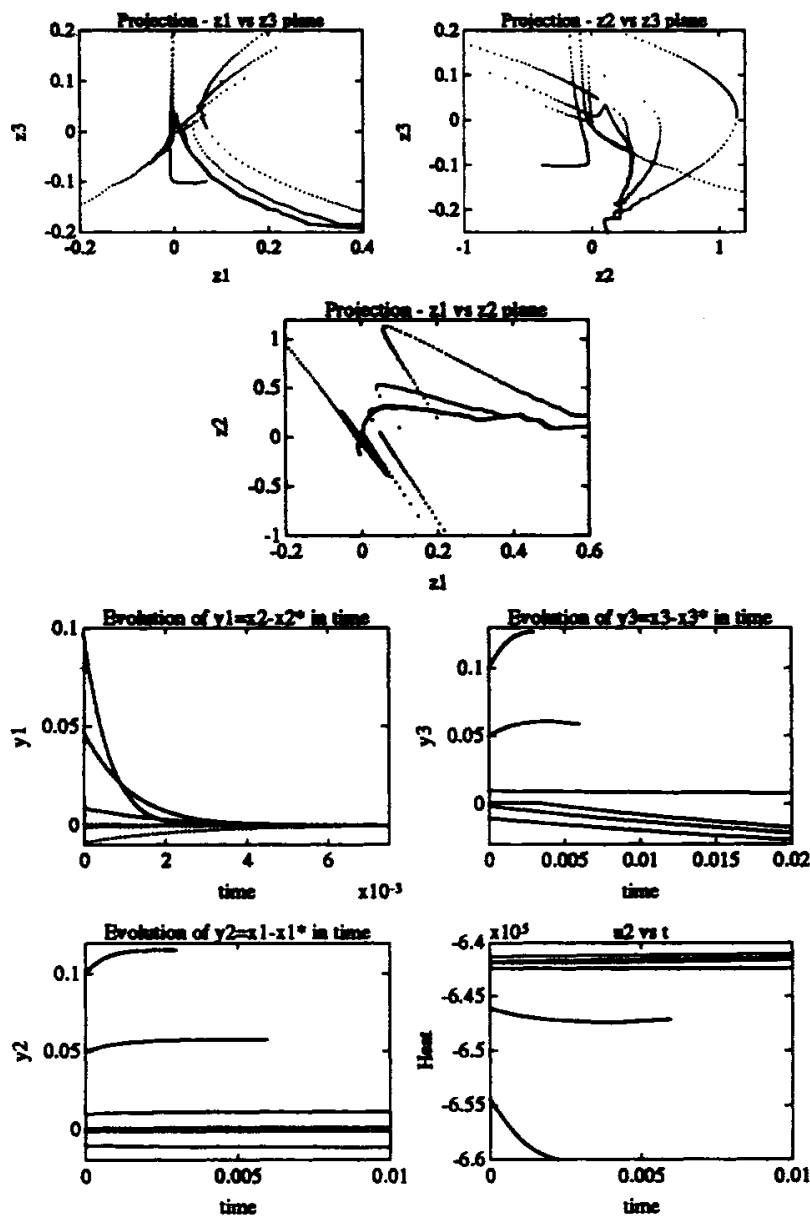

Thus, as neither one of the inputs, $u_{1}$ and $u_{2}$, is satisfactory for linearization of the input/output behavior of the system in the neighborhood of the optimum operating point, we tried to use both of them simultaneously: $u_{1}$ for linearization (because the system has a well-defined relative degree equal to 1 with respect to this input), and $u_{2}$ for local stabilization of the zero dynamics. The simulation results for the same set of parameters and $u_{i}^{*}=-332\left(u_{i}^{*}\right.$ is the value of $u_{2}$ at $x^{*}$ ) are shown below. We notice that there exists a considerably large neighborhood of the orizin for which the zero dynamics was made asymptotically stable. The operation around the optimum point is feasible in this case and both manipulated variables reach their equilibrium values for all trajectories starting "close" to the origin.
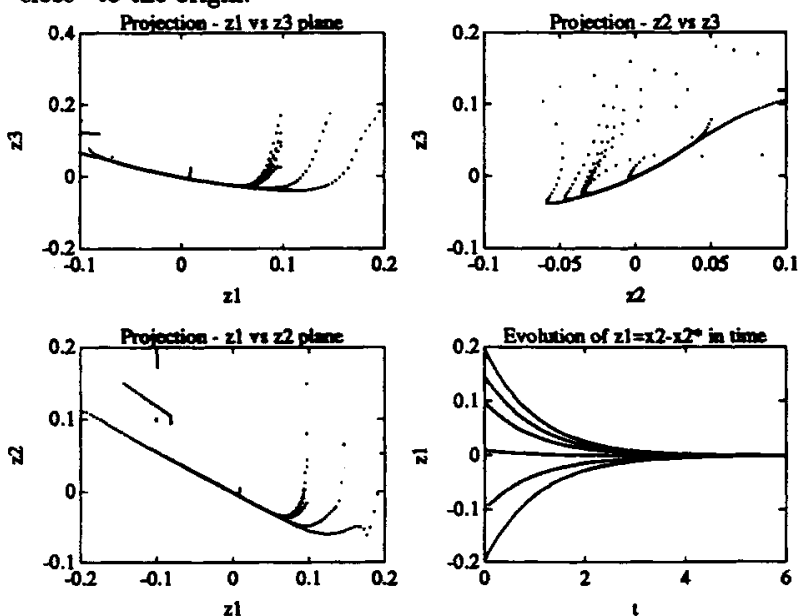
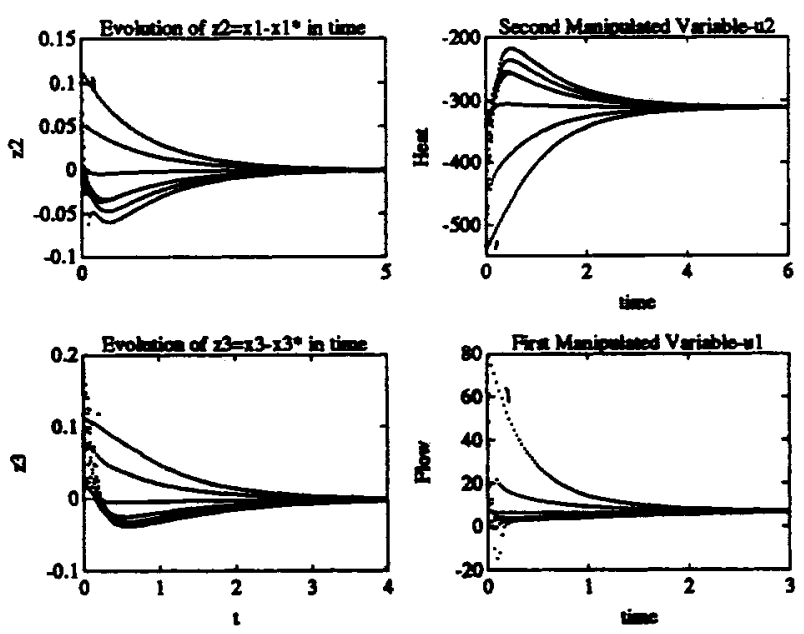

5 Conclusions

As a result of this work some control structures for nonlinear systems with unstable zero dynamics were developed.

The stable/anti-stable factorization approsch is an attempt of linearization of as much of the nonlinearities in the input/ontput map of the system as possible under the constraint that the resulting closed-loop system is stabilizable. Therefore it consists of an apprasch that establishes a compromise between exact linearization of the input/output behavior and local stabilization of the zero dynamics. Moreover, it seets an approximate decoupling of the stable and anti-stable parts of the zero dynamics such that the resulting feedback law uses only the first $r$ modes of the normal form, $(\xi)$, and the unstable modes of the zero dynamics, $\left(\eta_{1}\right)$. This makes the unobservable part of the resulting closed-loop system stable (if one neglects higher order terms).

The inner-outer factorization approach is a very straightforward procedure for the construction of a locally minimum-phase plant $P_{M}$ from the original plant $P$ by applying time reversal to the derivatives of the manipulated variable $u$. The resulting control architecture is analogous to the Smith Predictor for linear systems.

Finally, the multiple-input approach is an attempt of local stabilization of the internal dynamics by making use of extra degrees of freedom in the choice of the variables to be manipulated.

References

[1] C. Byrnes and A. Lsidori. Output regulation of nonlinear systems. IEEE Thenecetions on Automatic Control pages 131-140, 1990.

[2] J. Van der Vusee. Plug-flow type reactor versus tank reactor. Chem. Bry. Sci., pages 994-997, 1964.

(3) C. Economou, M. Morari, and O. Palsson. Internal model control. 5.ertension to nontinear systems. Ind. Engmg. Chem. Process Des. Dev., page 403, 1986.

[4] M. Fliess. Generalized controller canonical forms for linear and nonlinear dynamics. IBEE Thereactions on Axtomatic Control, pares 994-1001, 1990.

[5]C. Garcia and M. Morari. Internal model control. 1.a unifying review and some new results. Ind. Engng. Chem. Process Des. Dev., page 308, 1982.

[6] J. Hauser. Nonlinear control via uniform system approximation. In Proc. 29th CDC, 1990.

[7] A. Isidori. Nonlinear Control Systems. Springer-Verlag, 1989.

[8]C. Kravaris and C. Chung. Nonlinear feedback synthesis by global input/output linearization. AIChE J., page 592, 1987.

[9] C. Kravaris and P. Daoutidis. Nonlinear state feedback control of second-order nonminimum-phase nonlinear systems. Computers chem. Bagng., pages 439-449, 1990.

[10] A. Krener. Normal forms for linear and nonlinear systems. Contemp. Yath., 68, 1987.

[11]S. Ramanathan, R. Curl, and C. Kravaris. Dynamics and control of quasirational systems. $A K C h E J$., page $1017,1989$.

[12]S. Wiggins. Introduction to Applied Nonlinear Dracmical Systems and Cheos. Springer-Verlag, 1990.

[13] R. Wright and C. Kravaris. Nonminimum-phase compensation for nonlinear processes. AIChE J., pages 26-40, 1992.

[14] M. Zeits. Canonical forms for nonlinear systems. In IFAC Nonlinear Control Systems Design Proc., Capri, 1989. 